\title{
Piecewise adiabatic population transfer in a molecule via a wave packet
}

\author{
Evgeny A. Shapiro ${ }^{1}$, Avi Pe'er ${ }^{4}$, Jun Ye ${ }^{4}$, Moshe Shapiro ${ }^{1,2,3}$ \\ Departments of ${ }^{1}$ Chemistry and ${ }^{2}$ Physics, The University of British Columbia, Vancouver, V6T 1Z2, Canada, \\ ${ }^{3}$ Department of Chemical Physics, The Weizmann Institute, Rehovot, 76100, Israel, \\ ${ }^{4}$ JILA, National Institute of Standards and Technology and University of Colorado, Boulder, CO 80309-0440, USA.
}

(Dated: June 22, 2021)

\begin{abstract}
We propose a class of schemes for robust population transfer between quantum states that utilize trains of coherent pulses and represent a generalized adiabatic passage via a wave packet. We study piecewise Stimulated Raman Adiabatic Passage with pulse-to-pulse amplitude variation, and piecewise chirped Raman passage with pulse-to-pulse phase variation, implemented with an optical frequency comb. In the context of production of ultracold ground-state molecules, we show that with almost no knowledge of the excited potential, robust high-efficiency transfer is possible.
\end{abstract}

PACS numbers: 42.50.Hz, 82.50.Nd, 82.53.Kp, 34.50.Cx

With the advent of technologies that deal with quantum properties of matter, there is a need for methods of controlling quantum systems that are, on one hand, robust and conceptually simple, and on the other, flexible and applicable far beyond few-level arrangements. To address this need, our goal in this work is to utilize the intuition and control recipes of simple schemes for robust transfer of quantum population based on Adiabatic Passage (AP), and apply them to many-level quantum systems with complex dynamics. The key components of the method are coherent accumulation of amplitudes by a train of phase coherent optical pulses, and interference of quantum pathways in multi-state population transfer.

We test our ideas, simulating dynamics in KRb molecules, which is of special interest because of the prospect for creating deeply bound ultracold polar molecules starting from loosely bound Feshbach states [1, 2]. As translationally and vibrationally cold polar molecules are a prerequisite for many applications [3], their robust high-yield creation is a forefront goal.

Coherent accumulation has been studied in the context of perturbative control [4] and later on in precise spectroscopy using frequency combs [5]. Its use for complete population transfers was studied so far twice. One was the analysis within the 3 level model of piecewise adiabatic passage (PAP) 6]; the other study demonstrated population transfer through a wave packet with a coherent train of pump-dump pulses [7]. Here, we unite the two as special cases within one general framework, where the combination of adiabatic transfer concepts with a coherent train excitation (optical frequency comb) preserves the robustness of adiabatic transfer, but is applicable to composite quantum states.

The concept of piecewise adiabaticity is elucidated with two main examples. In piecewise stimulated Raman adiabatic passage (STIRAP), robust transfer is achieved through a slow variation of the intensity envelope of the driving pulse trains. In the other, which we term "piecewise chirped Raman passage" (CRP), robustness is obtained by slow variation of the excitation phase. We demonstrate how both examples can be extended to the case of the intermediate state being a wave packet, i.e. a multiplicity of intermediate states. In the spectral domain, the transfer is seen as a constructive interference of several AP pathways [8, 9. Last, we discuss the case of transfer without detailed knowledge of the intermediate dynamics. We show that by scanning the comb parameters of a train of unshaped pulse-pairs (repetition rate and intra-pair time separation) it is possible to achieve high efficiency transfer. The intermediate dynamics can then be studied using a two-dimensional mapping of the transfer efficiency as a function of the comb parameters.

A traditional AP transfers population from an initial to a target state by dressing the quantum system in slowly changing fields. If one of the time-dependent fielddressed eigenstates coincides with the initial state at the beginning of the process, and with the target at the end [9, 10, 11, 12, a complete and robust transfer is obtained as long as the dressing fields change adiabatically. Accordingly, the prescription of PAP [6] can be summarized as: For reference, consider any traditional AP, driven by slowly varying fields. Then, break the reference AP into a set of time intervals $\tau_{n}$, so short that only a small fraction of population is transferred between the eigenstates during each interval. Within each interval, the driving fields can be replaced by any other field amplitude shape, as long as the integral action of each of the fields over $\tau_{n}$ remains the same. For example, the smooth reference field can be replaced by a train of mutually coherent pulses. Last, vary the inter-pulse 'silent' time of the system's free evolution. Controlling the silent, free evolution durations and the phase of each of the driving fields allows control over the arising Ramsey-type interference picture.

Ref. 6] introduced piecewise versions of STIRAP [11, 12, which transfers population in a three-level system from state $|1\rangle$ into state $|3\rangle$ via the intermediate $|2\rangle$. In the key example, the field was given as a train of femtosecond "pump" and "dump" pulse pairs, resonantly coupling the eigenstates $|1\rangle$ and $|2\rangle$ (pump), and the eigenstates $|2\rangle$ and $|3\rangle$ (dump). The pulses - "kicks" 

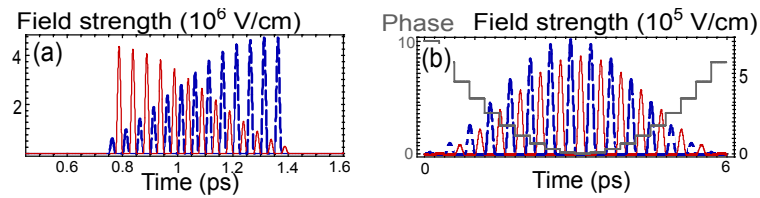

FIG. 1: (Color online). Sequences of femtosecond pulses in piecewise STIRAP (a) and piecewise CRP (b) between states $|1\rangle=3 S,|2\rangle=4 P$, and $|3\rangle=5 S$ of $\mathrm{Na}$ 13. Dashed blue: pump field envelope; solid red: dump field envelope; gray: the carrier phase in piecewise CRP with $\alpha_{P}=\alpha_{D}=0.2$.

- did not overlap in time and the pulse train envelopes were slowly varied to achieve PAP, as shown in Fig. 11(a).

Another conventional AP in a three-level system is CRP 11. The pump and dump pulses share a similar smooth temporal intensity profile; however, both pulses are frequency chirped, so that their phases are $\phi_{P, D}(t)=$ $\omega_{P, D} t+\alpha_{P, D}^{(\omega)}\left(t-t_{0}\right)^{2} / 2$. Like STIRAP, CRP transfers population from state $|1\rangle$ into $|3\rangle$ in a robust manner; unlike STIRAP, CRP creates transient population in state $|2\rangle$. For the piecewise version, all pulses are exactly on resonance. The effect of chirp is mimicked by varying the carrier phase of each pulse $\phi_{P, D}^{(0)}(n)=\phi_{P, D}(n, t)-\omega_{P, D} t$ quadratically with the pulse number $n$ :

$$
\phi_{P, D}^{(0)}(n)=\alpha_{P, D}\left(n-n_{0}\right)^{2} / 2
$$

as shown in Fig. 1(b). Pulse trains with such a piecewise chirp demonstrated piecewise adiabatic following in a two-state system [14]. Here we observe that the piecewise CRP in a three-level system is robust with respect to the field strengths, the number of pulse pairs, the values and signs of $\alpha_{P}$ and $\alpha_{D}$, and to additional delays between the pump and dump pulse trains.

In both foregoing examples, the details of the single pulse dynamics are not important, only the values of the basis state amplitudes after each $\tau_{n}$ matter. Based on this observation, below we extend the conceptual scheme of PAP, generalizing the eigenstate $|2\rangle$ to a multitude of states, forming a wave packet. This wave packet undergoes complex dynamics between the pairs of pump and dump kicks, but if the pulse repetition time coincides with a revival, the wave packet (almost) returns to its original state by the time the next pair of kicks arrives. The kicks, in turn, become rather complex operations implemented via an interplay of the shaped femtosecond pulses with the intermediate wave packet dynamics. We expect that, as long as the action of the kicks on the basis states $|1\rangle,|2\rangle$, and $|3\rangle$ mimics that of the dressing fields in the reference AP coarse-grained over $\tau_{n}$, the enforced population transfers in both schemes is insensitive to the parameters of the driving fields. Further, high fidelity of the revivals turns out to be unnecessary, as we demonstrate later with unshaped pulses.

Below we study this general idea simulating creation of deeply bound $X^{1} \Sigma^{+} \mathrm{KRb}$ molecules from a $a^{3} \Sigma^{+}$

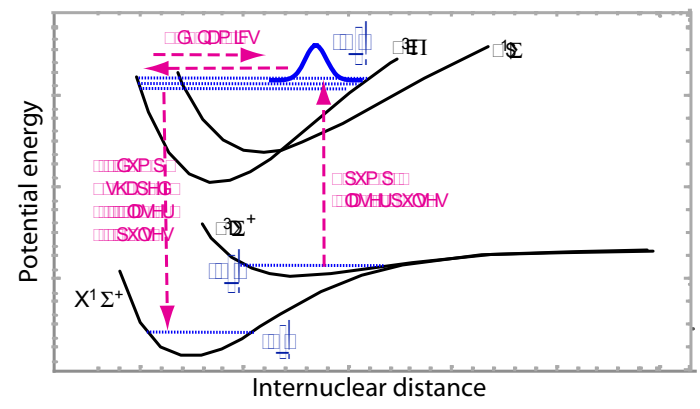

FIG. 2: (Color online). KRb potentials, basis states, and PAP arrangements discussed in the text.

state. The molecular potentials used in the simulations are shown schematically in Fig. 2. Eigenenergies and transition strengths are calculated using the algorithm FDEXTR [15] with the data input from Refs. [16, 17. The excited electronic state is modeled as the LS-coupled $A^{1} \Sigma$ and $b^{3} \Pi$ potentials. Although the model is too simple to precisely describe molecular dynamics on nanomicrosecond time scales, it retains the main complexity of the problem due to the coupled singlet-triplet evolution. We choose $|1\rangle$ to be the vibrational state $v=5$ $\left(E=-157 \mathrm{~cm}^{-1}\right)$ of the $a^{3} \Sigma^{+}$potential (Fig. 2). To trace the fidelity of the method, we also consider the amplitudes of vibrational states around the input state $(v=$ $0,1, \ldots, 12$ of the $a^{3} \Sigma^{+}$potential). The wave packet $|2\rangle$ is composed of up to 20 vibrational states of the LS coupled $A^{1} \Sigma-b^{3} \Pi$ potentials with the energies $E=11000-11400$ $\mathrm{cm}^{-1}$. State $|3\rangle$ is $v=22\left(E=-2490 \mathrm{~cm}^{-1}\right)$ of the ground $X^{1} \Sigma^{+}$potential; the neighboring $X^{1} \Sigma^{+}$states $v=16, \ldots, 28$ are included in the simulations as well.

The system, initially in state $|1\rangle$, is driven by a series of mutually coherent pump and dump femtosecond pulses. A single pump pulse (110 fs FWHM in intensity, $\sin ^{2} \alpha t$-shaped, as shown in Fig. 3(a)), excites an $A^{1} \Sigma-b^{3} \Pi$ wave packet, which oscillates on the coupled $A^{1} \Sigma-b^{3} \Pi$ potential surfaces with the singlet and triplet vibrational periods of the order of $800-900$ ps. After several vibrations the dynamics become irregular. However, at certain times, which correspond to two-surface revivals, the wave packet rephases with a fidelity reaching $\sim 0.9$. In our simulations, high-fidelity revivals were observed, roughly, every $80 \mathrm{ps}$; the particular revival time is sensitive to the spectroscopic data used as input. Following its excitation, the wave packet can be dumped into the single vibrational level $|3\rangle$. The shape of the femtosecond dump pulse is found by requiring that the wave packet before dumping overlaps well with the wave packet that would have been excited by the time-reversed dump, in a manner similar to the prescription of Ref. [7. The thus-found dump field is shown in Fig. 3(b).

The simulation results for both piecewise STIRAP and piecewise CRP are presented in Fig. 4. For STIRAP, shown in Fig. 4 (a,b), the field consists of 200 
Field strength

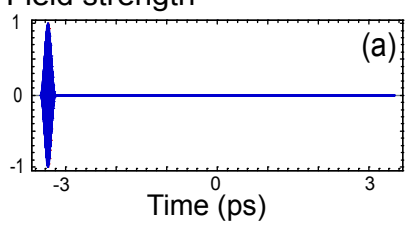

(a)

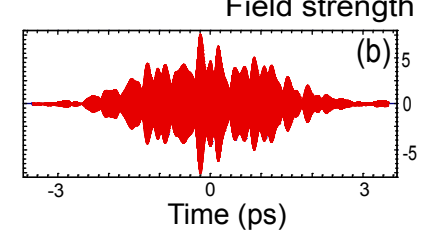

FIG. 3: (Color online) Time-dependent field of the pump (a), and dump (b) pulses. The field in each pulse is normalized relative to the maximum of the pump field.

pairs of pump and dump pulses, all shaped as in Fig. 3 . The trains envelopes vary linearly on the pulse number (Fig. 4(a)). The inter-pair separation is 1310.59 ps, which is close to a revival time of the wave packet $|2\rangle$. The carrier frequencies are chosen to match the Raman condition $\omega_{\text {Raman }}=\omega_{P}-\omega_{D}$. The carrier optical phase is kept constant throughout each pulse train by choosing the carrier frequency to coincide with a comb tooth [6]:

$$
\omega_{P, D}=2 \pi\left(N_{P, D} f_{r e p}+f_{0 P, D}\right),
$$

where $f_{\text {rep }}$ is the common repetition rate and $f_{0 P, D}$ is the carrier-envelope offset frequency in the pump (dump) train. The process is interferometrically sensitive to the inter-pulse delay $\left(f_{r e p}\right)$ and requires phase locking of the pump and dump combs; however, phase stabilization of frequency combs is now a standard technique [5].

If the spontaneous decay of the $A^{1} \Sigma-b^{3} \Pi$ states is neglected, the calculation predicts transferring $92 \%$ of population from $|1\rangle$ into $|3\rangle$. Introducing a $15-n s A^{1} \Sigma-b^{3} \Pi$ decay time reduces the transfer efficiency to $75 \%$. The maximum combined transient population of all the $A^{1} \Sigma$ $b^{3} \Pi$ levels reaches $\sim 0.04$. The transfer efficiency is found to be rather insensitive to the field strength profiles in the pulse trains, and varies within about $15 \%$ if the number of pulses is varied between 35 and 300 .

Figure 4(c,d) shows an example of CRP-like PAP. The field consists of 20 pairs of pump and dump pulses. The pump and dump pulse train envelopes are Gaussian (Fig. 4(c)). The inter-pair separation is 1309.93 ps. The central carrier phase in each field evolves with the pulse number according to Eq. (1), with $\alpha_{P}=\alpha_{D}=0.09$. The calculation with decaying $A^{1} \Sigma-b^{3} \Pi$ levels predicts transferring $60 \%$ of the initial population into the target state; neglecting the decay raises this number to $65 \%$. Approximately $2 \%$ of the population ends up distributed among eigenstates other than $|3\rangle$ of the target $X^{1} \Sigma^{+}$manifold; $22 \%$ of the initial population are transferred to neighboring states of $|1\rangle$ in the $a^{3} \Sigma^{+}$manifold at the end of the process. Unlike in the simple 3-level case, CRP-like transfer in the molecule is found to be significantly more sensitive than the STIRAP-like transfer to the number of pulses and the intensity profiles of the pump and dump pulse trains. We note that the required train envelope modulations (either in amplitude or phase) can be read-

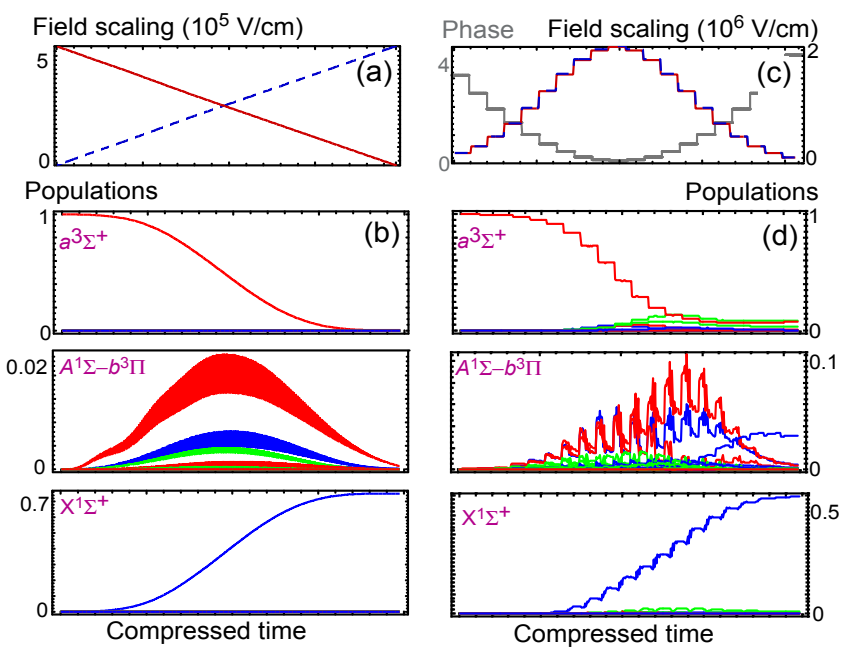

FIG. 4: (Color online) Dynamics of the STIRAP-like (a,b) and CRP-like (c,d) PAP in KRb. (a,c): Envelopes of the pump (dashed blue) and dump (red) pulses. Each point shows the factor by which the field shown in Fig. 3 is scaled. The carrier phase $\phi^{(0)}$ in the piecewise CRP, same for the pump and dump, is shown by the gray staircase in (c). (b,d): Population dynamics of all the levels in the system. Time is given at the "compressed" set of points: output is shown only for the times when the field is on.

ily generated experimentally by present modulators for standard 1-10 ns repetition-period pulse sources.

The dependence of the transfer efficiency on $\Delta T$ in both STIRAP-like and CRP-like PAP is irregular due to the complex dynamics of the $A^{1} \Sigma-b^{3} \Pi$ wave packet. Viewed from the spectral perspective, each of the $A^{1} \Sigma$ $b^{3} \Pi$ eigenstates can serve as an intermediate level for a separate AP pathway. Reminiscent of the traditional coherent control via a wave packet [9, 18, the AP pathways interfere to provide the population transfer into the target state. Thus the frequency comb source drives a coherently controlled AP process [5, 8, via the set of intermediate levels. Matching the repetition time and carrier phase to the wave packet revival time and phase ensures that all the possible AP pathways participate; spectral shaping of the pump and dump pulses sets constructive interference among the pathways.

Tailoring the optimal pulses requires detailed knowledge of the excited state structure, which is not always available. But what happens if the pulses are not shaped at all? Fig. 5(a) investigates the total efficiency of the STIRAP-like transfer under both pump and dump trains consisting of $50 \sin ^{2} \alpha t$-shaped pulses, with respect to the inter-pair delay $\Delta T=1 / f_{r e p}$ and intra-pair delay $\delta T$ between the pump and dump pulses. Raman resonance is maintained throughout the $f_{r e p}$ scan by varying the carrier-envelope frequency difference according to $f_{0 P}-f_{0 D}=\left(\omega_{\text {Raman }} / 2 \pi\right)-N f_{\text {rep }}$. The plot shows a remarkable landscape of vertical and horizontal features, 

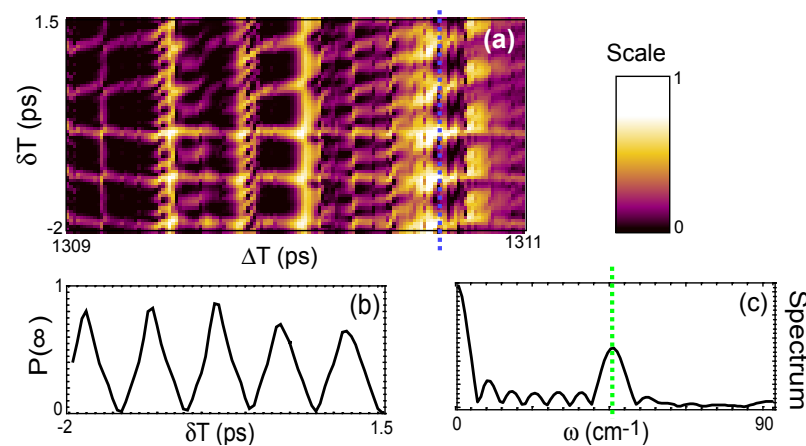

FIG. 5: (Color online)(a): An efficiency density plot for the STIRAP-like PAP with unshaped laser pulses in dependence on the inter-pair delay $\Delta T$ and the intra-pair delay $\delta T$. Positive $\delta T$ indicates that the pump comes after the dump; i.e. population remains in the excited wave packet until the next dump comes (suffering more spontaneous emission losses). (b): Vertical at $\Delta T=1310.62 \mathrm{ps}$ (blue dotted line in (a)). (c): Fourier transform of (b). The green dotted line marks the beat frequency between $E_{2}^{(1)}$ and $E_{2}^{(2)}$.

where some peaks reach $80 \%$ transfer efficiency. Note that no population is transferred to any of the $a^{3} \Sigma^{+}$or $X^{1} \Sigma^{+}$states, except $|1\rangle$ and $|3\rangle$, or to any of the intermediate states. In the spectral domain, either one state guides one of the interfering AP pathways, or it is simply missed by the narrow spectral selectivity of the frequency combs. In the time domain, destructive Ramsey-type interference prevents transitions to undesired levels.

To elucidate some of the structure observed in Fig. 5. consider $\delta T$ expressed in frequency as a linear spectral phase of the dump comb with respect to the pump comb. Accordingly, the phase of a specific Raman path at detuning $\Omega_{i}$ from the carrier frequency is $\varphi\left(\Omega_{i}\right)=$ $-\Omega_{i} \delta T+\phi_{d}\left(\Omega_{i}\right)$, where $\phi_{d}\left(\Omega_{i}\right)$ is the relative phase of the dipoles associated with this path. The vertical lines in the image correspond therefore to a $\Delta T$ value where one or several teeth are in one-photon resonance with Raman paths. Changing $\delta T$ at this $\Delta T$ reveals interference between these paths. A Fourier transform with respect to $\delta T$ reveals the spacing between the intermediate levels, as shown in Fig. 5(b,c), where mostly two Raman paths, via $E_{2}^{(1)}=11145 \mathrm{~cm}^{-1}$ and $E_{2}^{(2)}=11190 \mathrm{~cm}^{-1}$, contribute, and the Fourier transform of the transfer efficiency peaks at the beat frequency between $E_{2}^{(1)}$ and $E_{2}^{(2)}$. Horizontal lines, on the other hand, occur when $\delta T$ hits a special value, where $\varphi_{d}\left(\Omega_{i}\right) \approx \Omega_{i} \delta T$ for the participating Raman transitions; i.e., $\delta T$ is matched to the vibrational dynamics. In this case the transfer peaks even if there is no coherent accumulation in the intermediate levels, as was observed already in [7. Since the match to the dynamics is only approximate, the lines are not perfectly horizontal. In the best case scenario every dump pulse transfers into $|3\rangle$ exactly all the population excited to $|2\rangle$ from $|1\rangle$ by the preceding pump [7.

With some preliminary knowledge of the initial and target state's energies, the range of frequencies and the field strengths needed (but not of the exact values of intermediate energies and transition dipoles), one can take trains of unshaped pump and dump pulses, and scan $\Delta T$ and $\delta T$ to find efficient PAP transfers. In an analogy with 2D Fourier spectroscopy [19, a theoretical analysis of the 2D scan of the transfer efficiency may be able to provide the full spectroscopic information. Further, one can experimentally optimize the pulse shapes in order to maximize the transfer fidelity.

We thank V. Milner, I. Thanopulos, C. Koch, and S. Kotochigova for discussions and consultations. The work at JILA was supported by NIST and NSF.

[1] T. Köhler, K. Góral, P.S. Julienne Rev. Mod. Phys. 78, 1311 (2006), and references therein.

[2] J. J. Zirbel et.al., .Phys. Rev. Lett. 100143201 (2008).

[3] J. Doyle et. al., European Physical Journal D 31, 149 (2004), and references therein; A. Micheli, G.K. Brennen, P. Zoller, Nature Physics 2, 341 (2006).

[4] A. M. Weiner et. al., Science 247, 1317 (1990), L. Dhar, J.A. Rojers, K.A. Nelson, Chem. Rev. 94, 157 (1994).

[5] M.C. Stowe et. al., Adv. At. Mol. Opt. Phys. 55, 1 (2007); M.C. Stowe et. al., Phys. Rev. Lett 96, 153001 (2006); R. K. Shelton, et. al. Science 293, 1286 (2001).

[6] E.A. Shapiro et. al., Phys. Rev. Lett. 99, 033002 (2007).

[7] A. Pe'er et. al., Phys. Rev. Lett. 98, 113004 (2007).

[8] P. Kral, I.Thanopulos, M. Shapiro, Rev. Mod. Phys. 79, 53 (2007).

[9] M. Shapiro and P. Brumer, Principles of the Quantum Control of Molecular Processes (Wiley \& Sons, NY, 2003).

[10] S.A. Rice, M. Zhao, Optical control of molecular dynamics (John Wiley \& Sons, New York, 2000).

[11] J. Oreg, F.T. Hioe, J.H. Eberly, Phys. Rev. A 29, 690 (1984).

[12] U. Gaubatz et. al., J. Chem. Phys. 92, 5363 (1990); N.V. Vitanov et. al., Adv. At. Mol. Opt. Phys. 46, 55 (2001), and references therein.

[13] From NIST Atomic Spectra Database, (http://physics.nist.gov/PhysRefData/ASD/), we use $\mu_{12}=2.5$ a.u., and $\mu_{23}=3$ a.u.

[14] S. Zhdanovich et. al., Phys. Rev. Lett. 100, 103004 (2008).

[15] A.G. Abraskevich, D.G. Abraskevich, Computer Physics Communications 82, 193 (1994); ibid., 82, 209 (1994).

[16] S. Rousseau, A.R. Allouche, M. Aubert-Frecon, J. Mol. Spectrosc. 203, 235 (2000).

[17] S. Kotochigova, E. Tiesinga, P.S. Julienne, Eur. Phys. J. D 31189 (2004); S. Kotochigova, P.S. Julienne, E. Tiesinga, Phys. Rev. A 68022501 (2003).

[18] A. Stolow, Phil. Trans. Roy. Soc. A 356345 (1998).

[19] D. M. Jonas, Annu. Rev. Phys. Chem. 54, 425 (2003). 